\title{
Clinical Impact of Atrial Fibrillation in Patients With Hypertrophic Cardiomyopathy Results From Kochi RYOMA Study
}

\author{
Toru Kubo, MD; Hiroaki Kitaoka, MD; Makoto Okawa, MD; Takayoshi Hirota, MD; \\ Kayo Hayato, MD; Naohito Yamasaki, MD; Yoshihisa Matsumura, MD; \\ Toshikazu Yabe, MD; Jun Takata, MD; Yoshinori L. Doi, MD
}

\begin{abstract}
Background: There have been few studies of the clinical features of hypertrophic cardiomyopathy (HCM) in a community-based patient cohort in Japan.

Methods and Results: Cardiomyopathy registration was established in Kochi Prefecture and named the Kochi RYOMA (registry of myocardial diseases) study, consisting of 9 hospitals that registered 261 patients with a diagnosis of HCM. At registration, 74 patients $(28 \%)$ had documented paroxysmal or chronic atrial fibrillation (AF). Although most patients (93\%) were in New York Heart Association (NYHA) class I or II, 17 of the 18 patients in NYHA III had AF; 37 of the 74 patients with AF suffered from morbid events (embolism and/or heart failure (HF) admission), and 15 of 19 patients with embolic events had AF prior to or at the time of embolism. Of the 29 patients who had a history of HF admission, 8 had left ventricular systolic dysfunction, and the other 21 patients were hospitalized because of diastolic HF. AF occurred prior to HF in 20 of those 21 patients. Furthermore, 19 of those 20 patients with AF and diastolic HF were hospitalized within 1 year after detection of AF.

Conclusions: In an unselected regional registry, AF was the major determinant of clinical deteriorations in patients with HCM. (Circ J 2009; 73: 1599-1605)
\end{abstract}

Key Words: Atrial fibrillation; Embolism; Heart failure; Hypertrophic cardiomyopathy

$\mathbf{H}$ ypertrophic cardiomyopathy $(\mathrm{HCM})$ is a primary and genetically transmitted myocardial disorder with heterogeneous morphologic, functional, and clinical features. ${ }^{1-3}$ Since the initial description of HCM in 1958, death resulting from HCM, particularly when sudden, has been reported as largely confined to young persons. An annual mortality rate of 3-4\% has been reported from major tertiary HCM centers with highly selected referral patterns skewed toward high-risk patients. ${ }^{4-6}$ Recent observations in community-based and predominantly unselected patient cohorts in the USA and Europe suggest a more favorable prognosis than that suggested by the results of previous studies. ${ }^{7-10}$

\section{Editorial p 1589}

However, there have been few studies on the clinical features of HCM in a prospectively assembled, communitybased and unselected patient cohort in Japan. ${ }^{11}$ Therefore, we established the Kochi Cardiomyopathy Network, named the Kochi RYOMA (registry of myocardial diseases) study, to provide detailed descriptions of the clinical features of HCM in an unselected regional Japanese population. Here, we report on the clinical significance of HCM, particularly the impact of atrial fibrillation (AF), based on baseline characteristics at registration in the Kochi RYOMA study.

\section{Methods}

\section{Subjects}

The Kochi Cardiomyopathy Network consists of 9 hospitals serving as primary, secondary, and tertiary referral medical centers for cardiovascular patients in Kochi Prefecture, Japan, with 800,000 inhabitants. Between 2004 and 2008, 261 patients with a diagnosis of HCM were registered. The diagnosis of HCM was based on 2-dimensional (D) echocardiographic demonstration of unexplained left ventricular hypertrophy $(\mathrm{LVH})$. Informed consent was given by all subjects or their families in accordance with the Ethics Committee on Medical Research of Kochi Medical School.

\begin{abstract}
Clinical Evaluation
Evaluation of patients included medical history, clinical examination, 12-lead ECG, M-mode, 2-D and Doppler echocardiography, and ambulatory 24-h Holter ECG analysis. The severity and distribution of LVH were assessed in the parasternal short-axis plane at the mitral valve and papillary muscle levels. Left ventricular end-diastolic diameter (LVEDd) and end-systolic diameter (LVESd) were measured from M-mode and 2-D images obtained from parasternal long-axis views for calculation of fractional shortening $(\% \mathrm{FS}=(\mathrm{LVEDd}-\mathrm{LVESd}) / \mathrm{LVEDd} \times 100)$. The left ventricular (LV) outflow tract gradient was calculated from continu-
\end{abstract}

(Received March 1, 2009; revised manuscript received March 25, 2009; accepted April 2, 2009; released online July 9, 2009)

Department of Medicine and Geriatrics, Kochi Medical School, Nankoku, Japan

Mailing address: Hiroaki Kitaoka, MD, Department of Medicine and Geriatrics, Kochi Medical School, Oko-cho, Nankoku 783-8505, Japan.

E-mail: kitaokah@kochi-u.ac.jp

All rights are reserved to the Japanese Circulation Society. For permissions, please e-mail: cj@j-circ.or.jp 

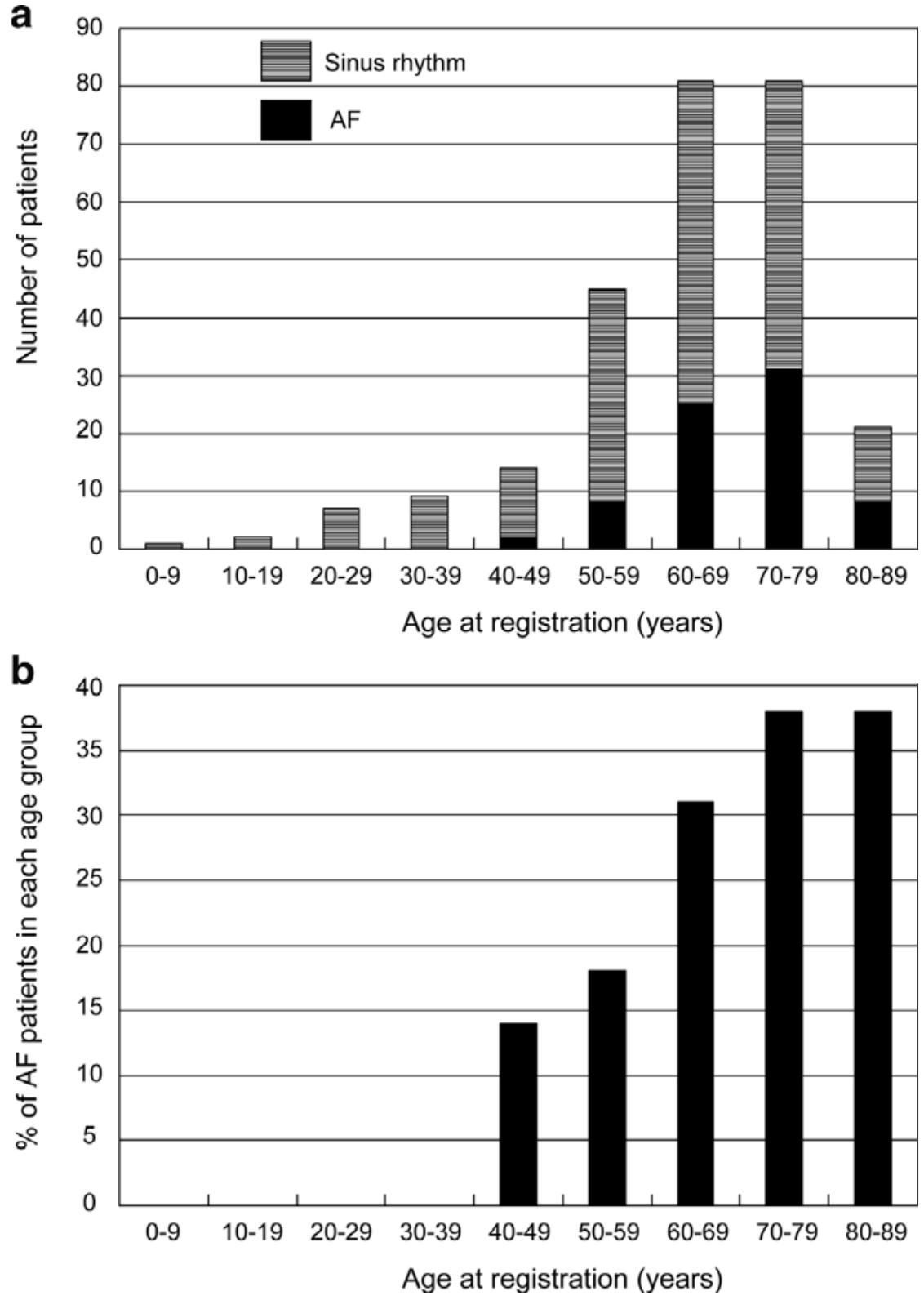

Figure 1. Age at registration. Number of patients (a) and percent of atrial fibrillation $(\mathrm{AF})$ in each age group (b). ous-wave Doppler using the simplified Bernoulli equation.

Based on morphologic and hemodynamic assessments by echocardiography, we divided the patients into the 5 groups: (1) hypertrophic obstructive cardiomyopathy (HOCM), defined as presence of basal LV outflow tract obstruction (gradient $\geq 30 \mathrm{mmHg}$ at rest), (2) midventricular obstruction (MVO), defined as presence of systolic LV cavity obliteration at the mid ventricle, creating MVO with a peak systolic gradient $\geq 30 \mathrm{mmHg}$ at rest, (3) dilated phase of HCM (D-HCM), defined as LV systolic dysfunction of the global ejection fraction $(\mathrm{EF})<50 \%$ (global $\mathrm{EF}$ was determined from apical 2- and 4-chamber views; concomitant coronary artery disease was excluded by coronary angiography and/or myocardial scintigraphy), (4) apical HCM, defined as hypertrophy confined to the LV apex, and (5) other (non-obstructive HCM other than D-HCM and apical HCM).

History of major morbid events including (1) hospitalization for heart failure (HF) and (2) embolic complications, including stroke and other systemic embolic events, which occurred as a result of probable or proven embolism was checked at registration.

\section{Data Analysis}

Statistical analysis was performed using SPSS (version 14.0) software (SPSS Inc, Chicago, IL, USA). All data are expressed as mean \pm SD (range) or frequency (percentage). Differences in continuous variables were assessed using Student's t-test. Pearson's chi-square test was used for comparisons between non-continuous variables, and Fisher's exact test was used when the expected frequency was lower than 5. Statistical significance was defined by $\mathrm{P} \leq 0.05$.

\section{Results}

\section{Clinical Characteristics at Registration}

The clinical characteristics of the 261 patients at HCM at registration are as follows. Age at registration and at diagnosis was $64 \pm 14$ (range: $9-88$ ) and $57 \pm 15$ (range: 6-87) years, 
Table. Clinical Characteristics of HCM Patients With AF at Registration

\begin{tabular}{|c|c|c|c|}
\hline & Patients with AF ( $\mathrm{n}=74)$ & Patients without $\mathrm{AF}$ ( $\mathrm{n}=187$ ) & $P$ value \\
\hline Age at registration, years & $69 \pm 9$ & $62 \pm 15$ & $<0.001$ \\
\hline Gender: male, $\mathrm{n}(\%)$ & $51(69 \%)$ & $122(65 \%)$ & 0.571 \\
\hline Age at diagnosis, years & $57 \pm 13$ & $56 \pm 16$ & 0.497 \\
\hline Reason for diagnosis: symptoms, $\mathrm{n}(\%)$ & $\begin{array}{c}57(77 \%) \\
\text { (embolic stroke in 4) }\end{array}$ & $\begin{array}{c}68(36 \%) \\
\text { (embolic stroke in 2) }\end{array}$ & $<0.001$ \\
\hline \multicolumn{4}{|l|}{ Symptoms at diagnosis, $\mathrm{n}(\%)$} \\
\hline Presence of symptoms & $60(81 \%)$ & $111(59 \%)$ & 0.001 \\
\hline Chest pain & $24(32 \%)$ & $55(29 \%)$ & 0.632 \\
\hline Palpitation & $31(42 \%)$ & $42(22 \%)$ & 0.002 \\
\hline Syncope & $6(8 \%)$ & $10(5 \%)$ & 0.401 \\
\hline \multicolumn{4}{|l|}{ NYHA functional class } \\
\hline I & $37(50 \%)$ & $125(67 \%)$ & 0.011 \\
\hline II & $28(38 \%)$ & $60(32 \%)$ & 0.376 \\
\hline III/IV & $9(12 \%)$ & $2(1 \%)$ & $<0.001$ \\
\hline \multicolumn{4}{|l|}{ Symptoms at registration, n (\%) } \\
\hline Presence of symptoms & $59(80 \%)$ & $106(57 \%)$ & 0.001 \\
\hline Chest pain & $18(24 \%)$ & $53(28 \%)$ & 0.511 \\
\hline Palpitation & $26(35 \%)$ & $35(19 \%)$ & 0.005 \\
\hline Syncope & $3(4 \%)$ & $8(4 \%)$ & 1.000 \\
\hline \multicolumn{4}{|l|}{ NYHA functional class } \\
\hline I & $26(35 \%)$ & $120(64 \%)$ & $<0.001$ \\
\hline II & $31(42 \%)$ & $66(35 \%)$ & 0.320 \\
\hline III/IV & $17(23 \%)$ & $1(1 \%)$ & $<0.001$ \\
\hline History of embolic complications, $\mathrm{n}(\%)$ & $15(20 \%)$ & $4(2 \%)$ & $<0.001$ \\
\hline History of heart failure admission, $\mathrm{n}(\%)$ & $27(36 \%)$ & $2(1 \%)$ & $<0.001$ \\
\hline Family history of HCM, n (\%) & $17(23 \%)$ & $47(25 \%)$ & 0.715 \\
\hline Family history of sudden death, $\mathrm{n}(\%)$ & $12(16 \%)$ & $33(18 \%)$ & 0.783 \\
\hline Pacemaker implantation, n (\%) & $12(16 \%)^{*}$ & $0(0 \%)$ & $<0.001$ \\
\hline ICD implantation, $\mathrm{n}(\%)$ & $2(3 \%)$ & $1(1 \%)$ & 0.195 \\
\hline \multicolumn{4}{|l|}{ Echocardiographic data at registration } \\
\hline \multicolumn{4}{|l|}{ Subtype, $\mathrm{n}(\%)$} \\
\hline HOCM & $8(11 \%)$ & $32(17 \%)$ & 0.203 \\
\hline MVO & $2(3 \%)$ & $5(3 \%)$ & 1.000 \\
\hline D-HCM & $8(11 \%)$ & $2(1 \%)$ & 0.001 \\
\hline Apical HCM & $8(11 \%)$ & $40(21 \%)$ & 0.047 \\
\hline Other & $48(65 \%)$ & $108(58 \%)$ & 0.291 \\
\hline Maximum LV wall thickness, mm & $18.7 \pm 3.6$ & $19.2 \pm 4.1$ & 0.379 \\
\hline LV end-diastolic diameter, mm & $48.8 \pm 6.3$ & $45.3 \pm 5.8$ & $<0.001$ \\
\hline Fractional shortening, $\%$ & $37.0 \pm 9.9$ & $42.5 \pm 7.5$ & $<0.001$ \\
\hline Left atrial diameter, $\mathrm{mm}$ & $50.8 \pm 7.1$ & $41.9 \pm 5.9$ & $<0.001$ \\
\hline
\end{tabular}

*Of 12 patients, 6 underwent pacemaker implantation for tachycardia-bradycardia syndrome, 2 had AF with bradycardia, 3 had complete atrioventricular block and 1 had cardiac resynchronization therapy.

HCM, hypertrophic cardiomyopathy; AF, atrial fibrillation; NYHA, New York Heart Association; ICD, implantable cardioverterdefibrillator; HOCM, hypertrophic obstructive cardiomyopathy; MVO, midventricular obstruction; D-HCM, dilated phase of hypertrophic cardiomyopathy; LV, left ventricular.

respectively, and 173 patients $(64 \%)$ were men. Approximately half $(48 \%)$ of the patients were diagnosed symptomatically; 6 were diagnosed as having HCM when embolic stroke occurred and 136 patients $(52 \%)$ were evaluated because of incidental findings, including ECG abnormalities and systolic murmur, and family screening. Symptoms was present in 171 patients $(66 \%)$ at diagnosis and in 165 patients (63\%) at registration; 64 patients $(25 \%)$ had proven familial $\mathrm{HCM}$ and 45 patients $(17 \%)$ had a family history of sudden death. At registration, 74 patients (28\%) had documented paroxysmal or chronic AF: 35 patients with paroxysmal AF defined as AF expected to convert to sinus rhythm within 7 days of onset, and 39 patients with chronic $\mathrm{AF}$ defined as persistent $\mathrm{AF}$ for more than 7 days from onset. Three patients received an implantable cardioverterdefibrillator ( 2 for ventricular fibrillation and 1 for sustained ventricular tachycardia). Of the 261 patients, there were 40 in the HOCM group, 7 in the MVO group, 10 in the D-HCM group, 46 in the apical HCM group, and 158 in the other group.

\section{Clinical Significance of AF}

Figure 1 shows the age distribution of the patients and the age-specific prevalence of $\mathrm{AF}$ (AF prevalence increased with age). The clinical characteristics of the patients with $\mathrm{AF}$ and without $\mathrm{AF}$ at registration are shown in Table. Patients with paroxysmal or chronic AF at registration were older than those without AF. Both at diagnosis and registration, patients with $\mathrm{AF}$ were more symptomatic (palpitation and dyspnea (New York Heart Association (NYHA) class $\geq$ II)). Furthermore, at registration, most patients (93\%) were in NYHA class I or II, and 17 of the 18 patients in NYHA III had AF. Of the 74 patients with AF, 37 (50\%) suffered from morbid events (embolism and/or HF admission). Embolic events occurred more frequently in patients with $\mathrm{AF}$ at registration than in patients without $\mathrm{AF}(\mathrm{P}<$ $0.001)$. In fact, 15 of 19 patients with embolic events (men=17) had AF prior to or at the time of the embolic event; the other 4 patients did not have documented AF despite the occurrence of embolism, and echocardiography revealed that 2 of them had apical aneurysm. Severe HF requiring hospitalization also occurred more frequently in 


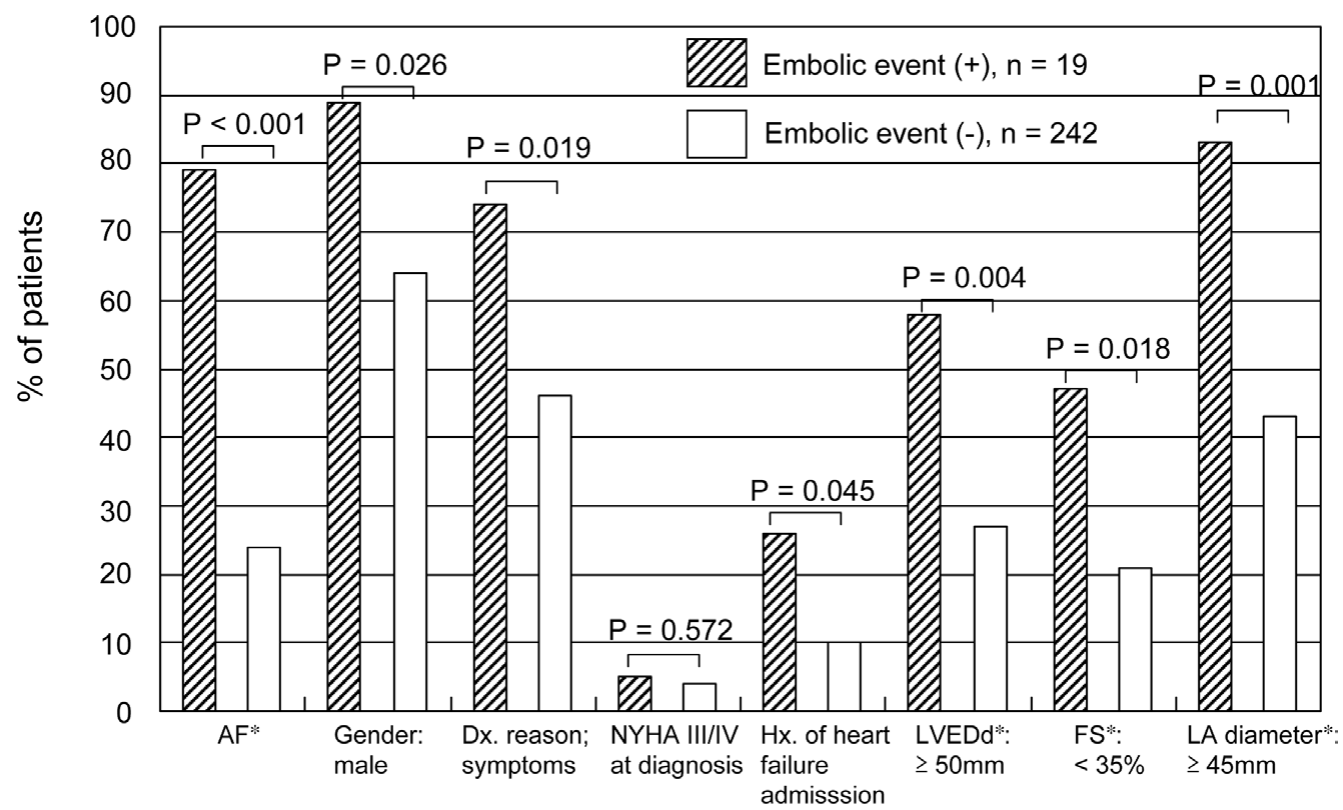

Figure 2. Clinical differences in patients who suffered from embolic events. AF, atrial fibrillation; Dx., diagnostic; NYHA, New York Heart Association functional class; Hx., history; LVEDd, left ventricular end-diastolic diameter; FS, fractional shortening; LA, left atrial. *Data at registration.

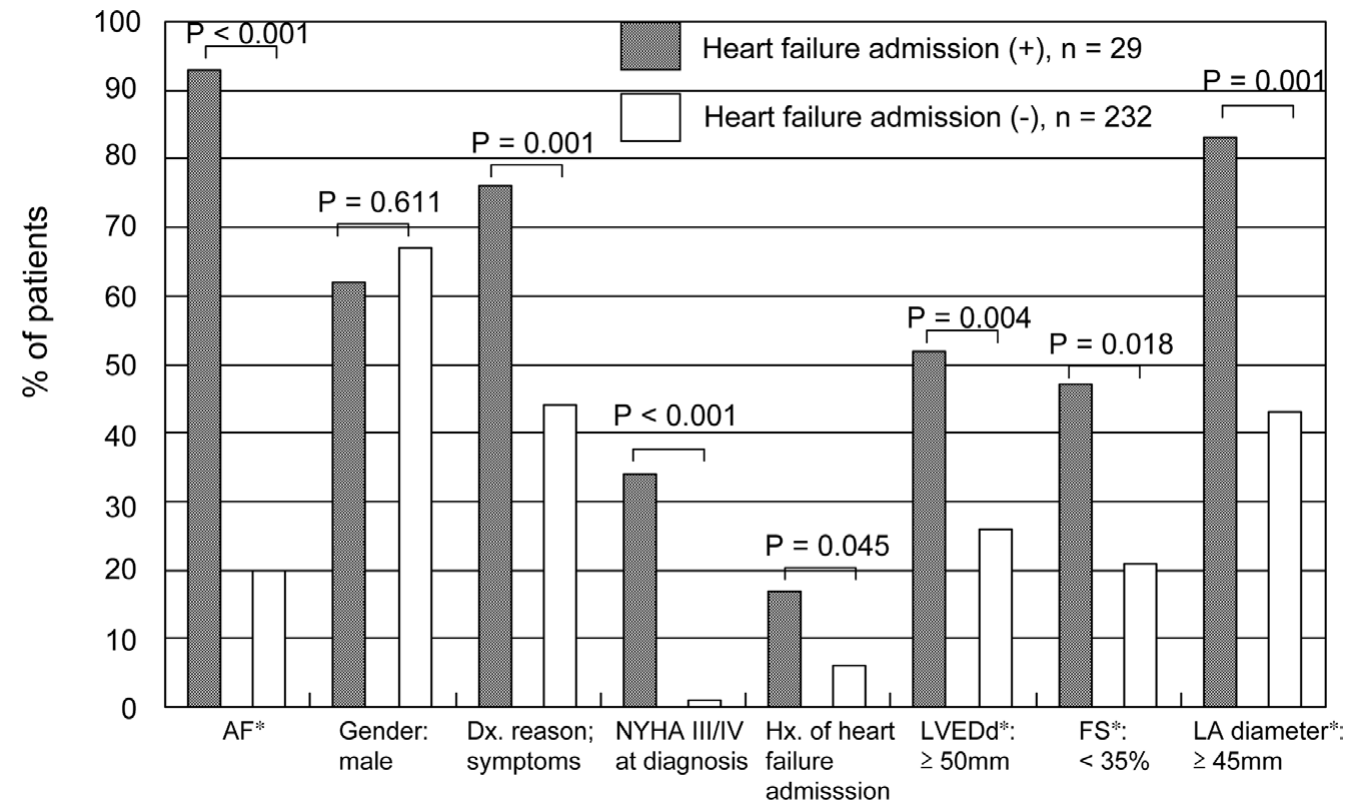

Figure 3. Clinical differences in patients who suffered from heart failure admission. AF, atrial fibrillation; Dx., diagnostic; NYHA, New York Heart Association functional class; Hx., history; LVEDd, left ventricular end-diastolic diameter; FS, fractional shortening; LA, left atrial. *Data at registration.

patients with $\mathrm{AF}(\mathrm{P}<0.001): 8$ of the 29 patients who had a history of HF admission had D-HCM, and the other 21 patients were hospitalized because of diastolic HF (AF occurred prior to diastolic HF in 20 of those 21 patients). Results of echocardiography at registration showed that LVEDd and left atrial diameters (LAd) were larger and $\% \mathrm{FS}$ was lower in patients with $\mathrm{AF}$ than in patients without AF. There were more D-HCM patients and fewer patients with apical HCM in the AF group.

Figures $\mathbf{2}$ and $\mathbf{3}$ show the clinical differences in patients who suffered from HCM-related morbid events (embolism or $\mathrm{HF}$ admission). In patients with these events, $\mathrm{AF}$ was more frequent and LVEDd and LAd were greater and FS lower at registration than in patients without events, and more patients with events were diagnosed as having symptomatic HCM than were patients without events. The 19 patients with cardioembolic events included more men than women. However, there was no gender difference in hospitalization for HF.

Distribution of ages at initial documentation of clinical complications is shown in Figure 4. AF, embolism, and $\mathrm{HF}$ admission occurred in patients over 40 years of age. 


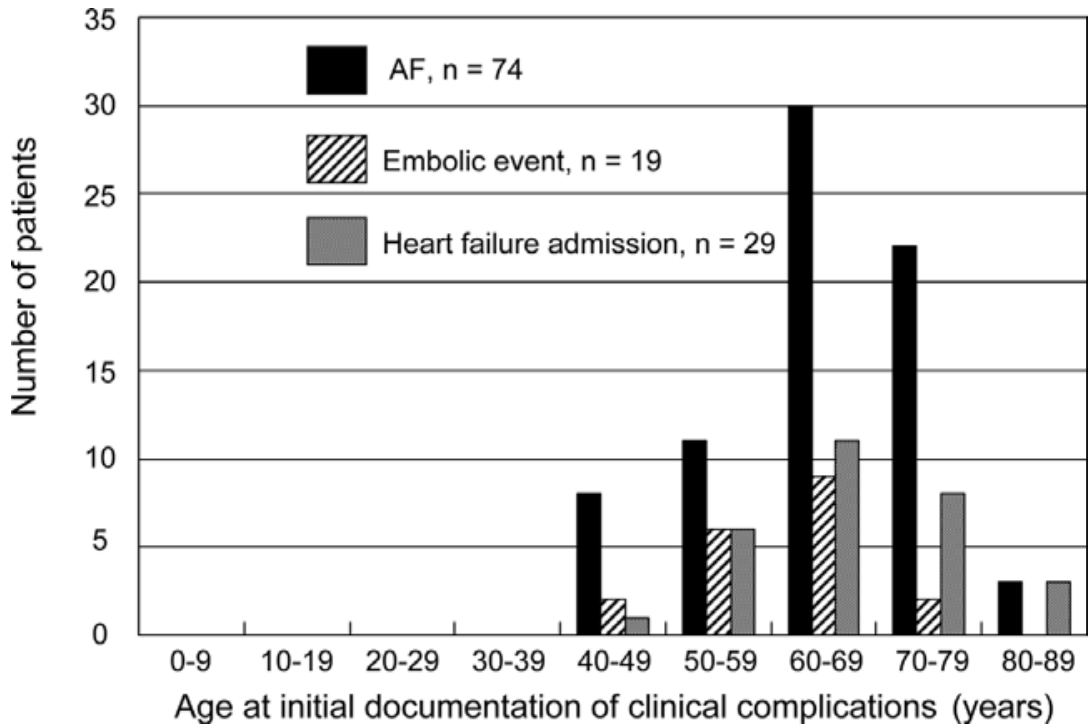

a

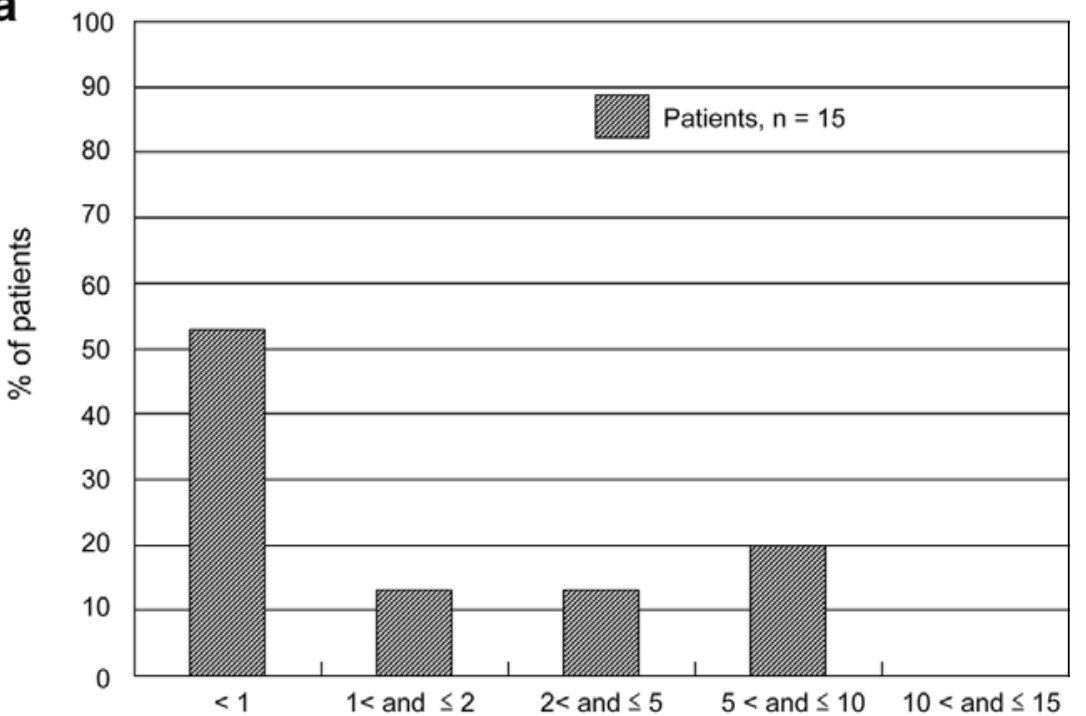

Period from AF detection to embolic events (years)

b

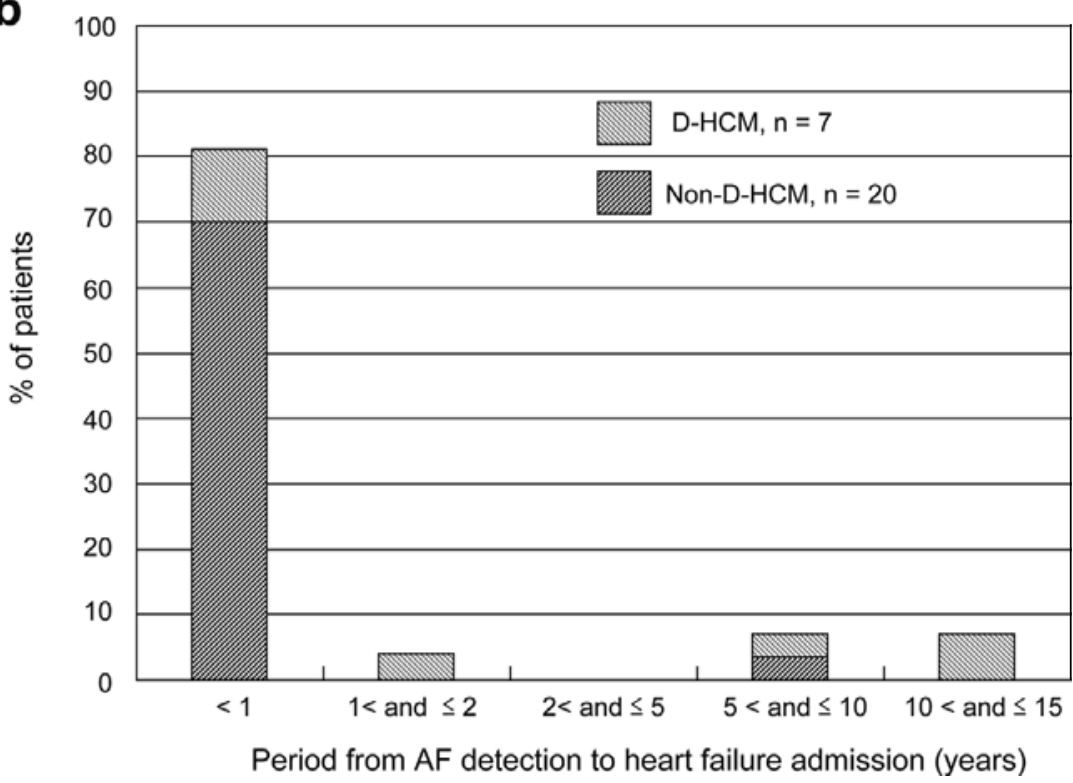

Figure 4. Age at initial documentation of clinical complications. AF, atrial fibrillation.
Figure 5. (a) Period from detection of atrial fibrillation (AF) to embolic events. (b) Period from $\mathrm{AF}$ detection to hospital admission because of heart failure. D-HCM, dilated phase of hypertrophic cardiomyopathy. 
The distribution of the ages of patients with these morbid events is similar to that at the time of initial documentation of AF. Figure 5 shows the period from detection of AF to embolism or HF admission. In the 15 patients with documented AF prior to embolism, embolic events occurred similarly in any period ( 8 patients had events within 1 year, but 4 of them were diagnosed as having HCM because of embolic events and AF was first detected at that time, indicating that these 4 patients might have suffered from $\mathrm{AF}$ for a long time). On the other hand, in the 27 patients with documented $\mathrm{AF}$ prior to $\mathrm{HF}$ admission, severe $\mathrm{HF}$ occurred within 1 year of detecting AF in most of the patients. The majority (95\%) of patients with preserved LV systolic function (non-D-HCM patients) were hospitalized for HF within 1 year.

\section{Discussion}

HCM is a primary myocardial disorder with heterogeneous clinical and morphologic features. ${ }^{1-3}$ Although most previous studies of this disease, based on populations from largely tertiary referral centers of highly selected patients, have shown a poor prognosis, largely because of sudden cardiac death, recent observations in community-based cohorts in Western countries suggest a more favorable prognosis than that indicated by the results of previous studies. ${ }^{4-10}$ The Kochi Cardiomyopathy Network (the Kochi RYOMA study) was established as a community-based and unselected Japanese patient cohort. In the present report, we provide detailed descriptions of the clinical features of an unselected regional HCM population according to the baseline characteristics at registration in the study. The impact of $\mathrm{AF}$ on major morbidity with cardioembolic events and HF admission was again highlighted, as in the previous limited number of existing studies on AF in $\mathrm{HCM}$ populations. ${ }^{12-17}$

\section{HCM Population}

Our regional cohort in the Kochi RYOMA study was older than HCM cohorts in previous studies, ${ }^{7-11,18}$ probably because Kochi Prefecture, where our study was performed, is located far from urban areas and is one of the most aged communities in Japan. The percentage of male patients in our cohort (64\%) was similar to the percentages in previous studies in Western countries and Japan. ${ }^{7-11,18}$ Although half of the patients were diagnosed because of symptoms, severe functional disability (NYHA class III/IV) was not common in our study. It is notable that embolic stroke was the first clinical manifestation leading to diagnosis of HCM in 6 patients (although ECG abnormalities had been detected in some of those patients, further evaluation had not been performed.). This number of patients was probably an underestimate because patients who died or had severe disability because of embolic events but without the diagnosis of HCM were not included and therefore there should be more patients who had stroke as the initial presentation of this disease. In clinical practice, routine screening by ECG and health education of the general population, as well as screening of HCM family members, plays an important role in preventing not only sudden cardiac death but also embolic stroke causing disability and even death. As for the subtypes of HCM, 40 patients (15\%) showed LV outflow obstruction (pressure gradient $\geq 30 \mathrm{mmHg}$ at rest), a prevalence that was lower than that in cohorts in Western countries. ${ }^{7-10,19}$

\section{Clinical Impact of AF}

The reported prevalence of AF in several HCM populations has been approximately $20 \%, 1,8,12,17,20$ whereas in the present study it was $28 \%$ and probably related to the aged cohort. In fact, the prevalence of AF increased with age. Our registration results showed that the prevalence of severe symptoms (NYHA class III/IV) and the frequency of morbid events, including embolism and HF admission, were significantly higher in patients with $\mathrm{AF}$ than in those without AF. Compared with the results from referral centers for HCM, there have been several studies showing that a significant portion of patients with HCM in regional cohort populations die from progressive HF and stroke in addition to premature sudden death. ${ }^{7,21} \mathrm{AF}$ seems to be an important factor for both HCM-related mortality and morbidity. In a recent study of Japanese AF patients, HF requiring hospitalization occurred at a rate of $2 \%$ per year and the existence of structural heart disease, including ischemic heart disease, valvular heart disease and cardiomyopathies, was the independent risk factor. ${ }^{22}$ The main pathophysiology of $\mathrm{HCM}$ is diastolic dysfunction with impaired filling because of abnormal relaxation and increased chamber stiffness, leading in turn to elevated LA and LV end-diastolic pressures. In most HCM patients, the occurrence of AF is considered to be a consequence of progressive diastolic dysfunction and LV remodeling, because in the present study the presence of AF was linked to advanced age, LA and LV enlargement and lower systolic function (\%FS).

In our study, the distribution of age at initial documentation of clinical complications showed that $\mathrm{AF}$, embolism and hospitalization for HF occurred similarly after middle age. However, there were different patterns in the period from detection of AF to morbid events, between cardioembolic complications and hospitalization for HF. Embolic events occurred equally at any time between 1 year to 10 years after $\mathrm{AF}$ onset, whereas the majority of patients who suffered from HF with preserved LV systolic function (ie, diastolic HF) were hospitalized within 1 year after the occurrence of AF. AF may be responsible for relatively acute progression of $\mathrm{HF}$, resulting from reduced diastolic filling and cardiac output as a consequence of loss of atrial contraction to LV filling and increased ventricular rate under conditions of LVH with preexisting impaired relaxation and compliance.

\section{Study Limitations}

We have presented the clinical features of HCM in a prospectively assembled, community-based and unselected patient cohort in a regional Japanese population, based on baseline characteristics at registration. Clinical complications including AF, embolic events, and hospitalization for HF were evaluated retrospectively. Furthermore, although morbid events occurred more frequently in patients with AF than in those without AF, half of the patients with AF did not suffer from morbid events. Therefore, further studies of the outcome, prognosis and prognostic factors in patients with HCM from the follow-up data in the Kochi RYOMA study are needed.

\section{Conclusions}

In our unselected regional registry, $\mathrm{AF}$ was the major determinant of clinical deterioration, including hospitalization for HF, as well as embolic events, in patients with HCM. Of note, diastolic HF within 1 year of the detection of $\mathrm{AF}$ 
was not uncommon.

\section{Acknowledgments}

Participating investigators from the study hospitals were Naohisa Hamashige, MD, Kazuya Kawai, MD, Masahiko Fukatani, MD, Shoichi Kubokawa, MD (Chikamori Hospital); Katsuhito Yamamoto, MD, Kazuhiko Sugimoto, MD (Kochi Health Sciences Center); Masaru Kimura, MD, Takashi Furuno, MD, Kiyoshi Nishino, MD (Japanese Red Cross Kochi Hospital); Takashi Yamasaki, MD (National Hospital Organization Kochi National Hospital); Masanori Kuwabara, MD (Tosa Municipal Hospital), Eisuke Yamada, MD, Eri Hoshikawa, MD (Susaki Kuroshio Hospital); Masafumi Akizawa, MD (Kochi Prefectural Aki Hospital); Fumiaki Kondo, MD, Naoki Onoda, MD (Kochi Prefectural Hata Kenmin Hospital); Hiromi Seo, MD (Kochi Medical School). We thank the physicians who made this study possible.

\section{References}

1. Spirito P, Seidman CE, McKenna WJ, Maron BJ. The management of hypertrophic cardiomyopathy. N Engl J Med 1997; 336: 775-785.

2. Maron BJ, McKenna WJ, Danielson GK, Kappenberger LJ, Kuhn HJ, Seidman CE, et al. American College of Cardiology/European Society of Cardiology clinical expert consensus document on hypertrophic cardiomyopathy: A report of the American College of Cardiology Foundation Task Force on Clinical Expert Consensus Documents and the European Society of Cardiology Committee for Practice Guidelines. J Am Coll Cardiol 2003; 42: 1687-1713.

3. Maron BJ. Hypertrophic cardiomyopathy: A systematic review. JAMA 2002; 287: $1308-1320$

4. McKenna WJ, Deanfield JE, Faruqui A, England D, Oakley MC, Goodwin JF. Prognosis in hypertrophic cardiomyopathy: Role of age and clinical, electrocardiographic and hemodynamic features. Am J Cardiol 1981; 47: 532-538.

5. Maron BJ, Savage DD, Wolfson JK, Epstein SE. Prognostic significance of 24 hour ambulatory electrocardiographic monitoring in patients with hypertrophic cardiomyopathy: A prospective study. Am J Cardiol 1981; 48: 252-257.

6. Seiler C, Hess OM, Schoenbeck M, Turina J, Jenni R, Turina M, et al Long-term follow-up of medical versus surgical therapy for hypertrophic cardiomyopathy: A retrospective study. J Am Coll Cardiol 1991; 17: $634-642$.

7. Cecchi F, Olivotto I, Montereggi A, Santoro G, Dolara A, Maron BJ Hypertrophic cardiomyopathy in Tuscany: Clinical course and outcome in an unselected regional population. $\mathrm{J} \mathrm{Am} \mathrm{Coll} \mathrm{Cardiol}$ 1995; 26: 1529-1536.

8. Maron BJ, Casey SA, Poliac LC, Gohman TE, Almquist AK, Aeppli DM. Clinical course of hypertrophic cardiomyopathy in a regional United States cohort. JAMA 1999; 281: 650-655.
9. Kofflard MJM, Ten Cate FJ, van der Lee C, van Domberg RT. Hypertrophic cardiomyopathy in a large community-based population: Clinical outcome and identification of risk factors for sudden cardiac death and clinical deterioration. J Am Coll Cardiol 2003; 41: $987-$ 993.

10. Maron BJ, Olivotto I, Spirito P, Casey SA, Bellone P, Gohman TE, et al. Epidemiology of hypertrophic cardiomyopathy-related death: Revisited in a large non-referral-based patient population. Circulation 2000; 102: 858-864.

11. Nasermoaddeli A, Miura K, Matsumori A, Sasayama Y, Morikawa Y, Kitabatake A, et al. Prognosis and prognostic factors in patients with hypertrophic cardiomyopathy in Japan: Results from a nationwide survey. Heart 2007; 93: 711-715.

12. Olivotto I, Cecchi F, Casey SA, Dolara A, Traverse JH, Maron BJ. Impact of atrial fibrillation on the clinical course of hypertrophic cardiomyopathy. Circulation 2001; 104: 2517-2524.

13. Glancy DL, O'Brien KP, Gold HK, Epstein SE. Atrial fibrillation in patients with idiopathic hypertrophic subaortic stenosis. Br Heart $J$ 1970; 32: 652-659.

14. Furlan AJ, Cracium AR, Raju NR, Hart N. Cerebrovascular complications associated with idiopathic hypertrophic subaortic stenosis. Stroke 1984; 15: 282-284.

15. Higashikawa M, Nakamura Y, Yoshida M, Kinoshita M. Incidence of ischemic strokes in hypertrophic cardiomyopathy is markedly increased if complicated by atrial fibrillation. Jpn Circ J 1997; 61: $673-681$.

16. Robinson K, Frenneaux MP, Stockins B, Karatasakis G, Poloniecki JD, McKenna WJ. Atrial fibrillation in hypertrophic cardiomyopathy: A longitudinal study. J Am Coll Cardiol 1990; 15: 1279-1285.

17. Maron BJ, Olivotto I, Bellone P, Conte MR, Cecchi F, Flygenring BP, et al. Clinical profile of stroke in 900 patients with hypertrophic cardiomyopathy. J Am Coll Cardiol 2002; 39: 301-307.

18. Miura K, Nakagawa H, Morikawa Y, Sasayama S, Matsumori A, Hasegawa K, et al. Epidemiology of idiopathic cardiomyopathy in Japan: Results from a nationwide survey. Heart 2002; 87: 126-130.

19. Maron MS, Olivotto I, Zenovich AG, Link MS, Pandian NG, Kuvin JT, et al. Hypertrophic cardiomyopathy is predominantly a disease of left ventricular outflow tract obstruction. Circulation 2006; 114: $2232-2239$.

20. Nagai T, Ogimoto A, Okayama H, Ohtsuka T, Shigematsu Y, Hamada $\mathrm{M}$, et al. A985G polymorphism of the endothelin-2 gene and atrial fibrillation in patients with hypertrophic cardiomyopathy. Circ J 2007; 71: $1932-1936$.

21. Kitaoka H, Kubo T, Okawa M, Hitomi N, Furuno T, Doi YL. Left ventricular remodeling of hypertrophic cardiomyopathy: Longitudinal observation in a rural community. Circ J 2006; 70: 1543-1549.

22. Fukuda T, Yamashita T, Sagara K, Kato T, Sawada H, Aizawa T. Development of congestive heart failure in Japanese patients with atrial fibrillation. Circ J 2007; 71: 308-312. 